\title{
ON THE MODULUS OF BOUNDARY VALUES OF HOLOMORPHIC FUNCTIONS
}

\author{
R. MICHAEL RANGE ${ }^{1}$
}

\begin{abstract}
A differential geometric method is introduced to study the modulus of boundary values of holomorphic functions on smoothly bounded pseudoconvex domains $D$ in $\mathbf{C}^{n}, n>2$. It is shown that functions in $A(D)$ are determined up to a constant factor by their modulus on an open subset of the Shilov boundary. For the case of $H^{\infty}(D)$, it is shown that inner functions which satisfy a certain local condition are constant.
\end{abstract}

0 . Introduction. Let $D$ be a bounded domain in $\mathbf{C}^{n}$ and let $A(D)$ denote the Banach algebra of continuous functions on $\bar{D}$ which are holomorphic on the interior of $\bar{D}$. The Shilov boundary of $A(D)$, denoted by $S(D)$, is the smallest closed subset of $\partial D$ such that $\|f\|=\sup \{|f(z)|: z \in S(D)\}$ for all $f \in$ $A(D)$. A function $f \in A(D)$ is called inner if $|f|=1$ on the Shilov boundary $S(D)$.

In dimension one there are many inner functions in $A(D)$; similarly, on the unit polydisc $\Delta^{n}=\left\{z \in \mathbf{C}^{n}:\left|z_{j}\right|<1\right.$ for $\left.j=1, \ldots, n\right\}$ with Shilov boundary $S\left(\Delta^{n}\right)=\left\{z \in \mathbf{C}^{n}:\left|z_{j}\right|=1\right.$ for $\left.j=1, \ldots, n\right\}$ there are many nontrivial inner functions. However, as is well known, on the ball, or more generally, on strictly pseudoconvex domains $D \subset \mathbf{C}^{n}$, if $n \geqslant 2$, inner functions in $A(D)$ are constant. In fact, in this case $S(D)=\partial D$; if $f \in A(D)$ with $\|f\|=1$ is not constant, there is $c \in \mathbf{C},|c|<1$, such that $X=\{z \in D: f(z)=c\}$ is not empty; since $n \geqslant 2, X$ is an analytic variety of positive dimension whose closure must meet $\partial D$, and hence $|f|<1$ somewhere on $\partial D$.

The main result of this paper is a generalization of this last result to arbitrary pseudoconvex domains $D$ with smooth boundary in the following stronger form: If $f, g \in A(D)$ and $|f|=|g|$ on some (relatively) open subset of the Shilov boundary $S(D)$, then $f=e^{i \alpha} g$ for some $\alpha \in \mathbf{R}$.

A weaker result was announced by Debiard and Gaveau [1]: If $|f|=|g|$ on $\partial D$, then $f=e^{i \alpha} g$. Their method is potential theoretic and uses certain global metrics introduced by $P$. Malliavin.

In contrast, the method presented here is local differential geometric; it exhibits the role of the complex structure and convexity carried by the

Received by the editors September 24, 1976 and, in revised form, November 22, 1976.

AMS (MOS) subject classifications (1970). Primary 32E25, 46J15; Secondary 32F15, 46J10, 46J20, 53B99.

Key words and phrases. Shilov boundary, pseudoconvex domain, inner function, point of finite type, boundary values of holomorphic functions, $C R$-manifold, $C R$-function.

${ }^{1}$ Author partially supported by NSF grant MCS75-07062 A01.

- American Mathematical Society 1977 
boundary of domains in $\mathbf{C}^{n}, n \geqslant 2$, and it helps to explain the differences in the examples above. Moreover, the method can be adapted to different situations; for example, one obtains a local condition for an $H^{\infty}$ inner function which forces it to be constant (cf. §3).

1. $C R$-functions of constant modulus. The natural setting for the local differential geometric method discussed in this paper is in the context of $C R$-manifolds and $C R$-functions (for definitions, etc., see, for example [9]). Let $M$ be a $C R$-submanifold of an open set $U \subset C^{n}$. Denote by $T^{1,0}(M)$ the subbundle of the complexified tangent bundle $C T M$, whose fiber $T_{P}^{1,0}(M)$ at a point $P \in M$ consists of those tangent vectors which are of type $(1,0)$ in the ambient space $C^{n}$. Let $C^{\mathcal{T}_{P}}(M)$ and $\mathcal{T}_{P}^{1,0}$ be the set of germs at $P$ of $C^{\infty}$ sections of the respective bundles. $C \mathcal{T}_{P}(M)$ carries a natural Lie algebra structure. Denote by $\mathcal{L}_{P}(M)$ the Lie subalgebra generated by $\mathscr{T}_{P}^{1,0} \oplus \widetilde{\mathscr{T}}_{P}^{1,0}$ in C $\mathcal{T}_{P}(M)$.

Definition 1. The point $P \in M$ is called of finite type if the evaluation map $\mathcal{L}_{P}(M) \rightarrow \mathbf{C} T_{P}(M)$ is onto.

REMARK 1. If $P \in M$ is of finite type, so are all points in $M$ near $P$.

REMARK 2. If $M$ is a hypersurface, for $P$ to be of finite type it is sufficient that the Leviform of $M$ has at least one nonzero eigenvalue at $P$, or more generally, that there is $L \in \mathfrak{T}_{P}^{1,0}$ of finite order at $P$ in the sense of Kohn [5]. If $M$ is a hypersurface in $\mathbf{C}^{2}$, the above definition coincides with Definition 2.3 of Kohn [4].

REMARK 3. As far as this paper is concerned, the exceptional role of the one-dimensional case comes from the fact that there are no points of finite type on the boundary of domains $D \subset \mathbf{C}$.

As we will consider continuous and $L^{\infty}$-boundary values of holomorphic functions, the concept of a $C R$-function should properly be formulated in the language of currents. In order to avoid much notation, we give a simpler definition. For a submanifold $M$ of $U \subset \mathbf{R}^{n}$ we denote the induced Lebesgue measure on $M$ by $d m$.

Definition 2. Let $M$ be a CR-submanifold of an open set $U \subset C^{n}$. A complex valued function $f \in L_{\text {loc }}^{1}(M, d m)$ is a $C R$-function at $P \in M$ if $\bar{L} f=0$ near $P$ in the weak sense for all $L \in \mathcal{T}_{P}^{1,0}$, i.e. for each such $L$ there is a neighborhood $Y$ of $P$ in $M$ such that $L$ is defined on $Y$ and $\int f(\bar{L} \phi) d m=0$ for all $\phi \in C_{0}^{\infty}(Y)$. $f$ is a $C R$-function on $M$ if $f$ is a $C R$-function at every $P \in M$.

LEMMA 1. Let $M$ be a CR-submanifold of an open set $U \subset \mathbf{C}^{n}$ and let $P \in M$ be of finite type. Suppose $f \in L_{\text {loc }}^{1}(M, d m)$ satisfies $L f=\bar{L} f=0$ near $P$ for all $L \in \mathcal{T}_{P}^{1,0}$. Then $f=$ constant almost everywhere $(\mathrm{dm})$ in a neighborhood of $P$.

Proof. The hypothesis about $f$ implies that $X f=0$ near $P$ for all $X \in$ $\mathcal{L}_{P}(M)$. Since $P$ is of finite type, finitely many $X_{1}, \ldots, X_{s} \in \mathcal{L}_{P}(M)$ will induce a basis for $\mathrm{CT}_{\zeta}(M)$ at all points $\zeta$ in a neighborhood of $P$. Thus $d f=0$ near $P$ in the weak sense, and the lemma follows. 
Proposition Let $f \in C^{1}(M)$ be a CR-function at $P \in M$. Suppose $P$ is of finite type and $|f|$ is constant in a neighborhood of $P$. Then $f$ is constant near $P$.

Proof. The proof imitates the well-known argument for the corresponding statement about holomorphic functions. Choose a neighborhood $W$ of $P$ such that $f$ is $C R$ and $|f|=c, c \in \mathbf{R}$, on $W \cap M$. If $c=0$, there is nothing to prove; so assume $c \neq 0$. For $L \in \mathscr{T}_{P}^{1,0}$ one has $0=L\left(|f|^{2}\right)=(L f) \bar{f}$, since $L \bar{f}=(\bar{L} f)=0$. As $\bar{f} \neq 0$, it follows that also $L f=0$ near $P$, and the conclusion follows from Lemma 1.

2. Continuous boundary values. The proof of the Proposition in $\$ 1$ does not make sense for functions which are $C R$ only in the weak sense. However, by modifying the argument, one can handle the following important case.

Lemma 2. Suppose $M$ is a hypersurface in an open set $U \subset \mathbf{C}^{n}$, such that $U-M$ consists of two connected components $U^{+}$and $U^{-}$. Let $P \in M$ be of finite type (this implies $n \geqslant 2$ !). Let $f \in A\left(U^{+}\right)$and suppose that there is a neighborhood $W \subset U$ of $P$ such that $|f|$ is constant on $W \cap M$. Then $f$ is constant on $U^{+}$.

Proof. It is enough to show that $f=c \in \mathbf{C}$ on a nonempty open subset of $M$. In fact, if $f-c=0$ on $V \cap M \neq \varnothing, V$ open in $\mathbf{C}^{n}$, then $f-c=0$ on a component of $V \cap U^{+}$, and hence on $U^{+}$; this fact can be proved, for example, by using the well-known one-dimensional case (cf. [7, Theorem 11.22] for the case of the unit disc in C) and Fubini's Theorem.

The case $|f|=0$ on' $^{\prime} W \cap M$ being trivial, one may assume without loss of generality that $|f|=1$ there. By continuity there is a neighborhood $V \subset W$ of $P$, such that $|f| \geqslant 1 / 2$ on $V \cap U^{+}$. Hence $f$ and $f^{-1} \in A\left(V \cap U^{+}\right)$, and their restrictions to $V \cap M$ are continuous $C R$-functions, i.e. $\bar{L} f=\bar{L}\left(f^{-1}\right)=$ 0 near $P$ for all $L \in \mathfrak{T}_{P}^{1,0}(M)$. Since $f^{-1}=\bar{f}$ on $V \cap M$ and $P$ is of finite type, the hypotheses of Lemma 1 are satisfied; it follows that $f$ is constant on a neighborhood of $P$ in $M$. Q.E.D.

THEOREM I. Let $D \subset \mathbf{C}^{n}, n \geqslant 2$, be a bounded pseudoconvex domain with smooth boundary. Let $f, g \in A(D)$ and suppose that there is a relatively open nonempty subset $Y$ of the Shilov boundary $S(D)$, such that $|f|=|g|$ on $Y$. Then $f=e^{i \alpha} g$ for some $\alpha \in \mathbf{R}$.

Proof. We first show that one can replace $Y$ by a set $Y^{*} \subset Y$ which is open in $\partial D$, and such that every point $P \in Y^{*}$ is of finite type. By Hakim and Sibony [2, Theorem 1], $S(D)$ is the closure of the set of strictly pseudoconvex points in $\partial D$. Thus $Y$ contains a strictly pseudoconvex point $P$. Choose an open neighborhood $U$ of $P$ in $\mathbf{C}^{n}$, such that $\partial D$ is strictly pseudoconvex at every point in $U \cap \partial D$. Again, by the theorem of Hakim and Sibony, $U \cap \partial D \subset S(D)$; it follows that $Y^{*}=Y \cap U$ has all the required properties.

Next, it is enough to show that $f=e^{i \alpha} g$ on some nonempty open subset of 
$D$. In case $|f|=|g|=0$ on $Y^{*}$, this holds trivially. So one may assume that there is $Q \in Y^{*}$ such that $|f(Q)|=\mid g(Q) \neq 0$. By continuity, there are a constant $\delta>0$ and an open neighborhood $V$ of $Q$, such that $V \cap \partial D \subset Y^{*}$ and $|f(z)|,|g(z)| \geqslant \delta$ for $z \in V \cap D$. It follows that $f / g \in A(D \cap V)$ and $|f / g|=1$ on $V \cap \partial D$; by Lemma $2, f / g=e^{i \alpha}$ on $V \cap D$ for some $\alpha \in \mathbf{R}$. Q.E.D.

3. Inner functions in $H^{\infty}(D)$. Let $D \subset \mathbf{C}^{n}$ be a bounded domain with smooth boundary and denote by $H^{\infty}(D)$ the algebra of bounded holomorphic functions on $D$. By Fatou's Theorem (cf. Stein [8]), $f \in H^{\infty}(D)$ has nontangential limits almost everywhere on $\partial D$. Each $f \in H^{\infty}(D)$ thus defines a boundary value function in $L^{\infty}(\partial D)$, denoted by $f^{*}$, which is a $C R$-function on $\partial D$. Unfortunately the proof of Lemma 2 cannot be applied to $f \in$ $H^{\infty}(D)$ with $\left|f^{*}\right|=1$, a.e. on some open subset of $\partial D$. However, one obtains the following result.

TheOREM II. Let $D \subset \mathbf{C}^{n}, n \geqslant 2$, be a bounded pseudoconvex domain with smooth boundary. Suppose that $f \in H^{\infty}(D),\|f\| \leqslant 1$, satisfies $\left|f^{*}\right|=1$ a.e. on a nonempty open subset $Y$ of the Shilov boundary $S(D)$ of $A(D)$, and that there is a $P \in Y$ such that the cluster set $\mathrm{Cl}(f, P)$ of $f$ at $P$ is not equal to the closed unit disc $\bar{\Delta}$. Then $f$ is constant.

Proof. By composing $f$ with a suitable automorphism of the unit disc one may assume without loss of generality that $0 \notin \mathrm{Cl}(f, P)$, i.e. there are a constant $\delta>0$ and an open neighborhood $W$ of $P$ such that $|f| \geqslant \delta$ on $W \cap D$. Thus $f$ and $f^{-1} \in H^{\infty}(W \cap D)$. As in the proof of Theorem I, one may assume that $Y \subset W, Y$ is open in $\partial D$ and each point of $Y$ is of finite type. The conclusion now follows as in the proof of Lemma 2; the argument at the beginning of that proof works just as well for $f \in H^{\infty}(W \cap D)$.

Corollary. $^{2}$ Let $D \subset \mathbf{C}^{n}, n \geqslant 2$, be a domain with smooth strictly pseudoconvex boundary. Then an inner function in $H^{\infty}(D)$ is either constant or else its cluster set at every point $P \in \partial D$ is the closed unit disc.

RemarK 4 . On a strictly pseudoconvex domain $D$ the cluster set at $P \in \partial D$ of $f \in H^{\infty}(D)$ is equal to the range of the Gelfand transform of $f$ on the fiber $\Re_{P}$ over $P$ of the maximal ideal space $\Re$ of $H^{\infty}(D)[6]$.

REMARK 5. In dimension 1 there are inner functions which satisfy the above cluster value condition. In fact, it follows from Hoffman [3, pp. 68-69], that for a given inner function $f \in H^{\infty}(\Delta)$ and $P \in \partial \Delta$, either $\mathrm{Cl}(f, P)=\bar{\Delta}$ or $f$ extends analytically across $P$. Thus, any Blaschke product whose zeroes cluster everywhere on $\partial \Delta$ will do.

\footnotetext{
${ }^{2}$ After completion of this manuscript the author learned that D. Marshall and N. Varopoulos, and also W. Rudin, had obtained the corollary for the case of the unit ball $B$. Their method is based on restricting $f$ to suitably chosen analytic discs with boundary contained in $\partial B$.
} 


\section{REFERENCES}

1. A. Debiard and B. Gaveau, Démonstration d'une conjecture de H. Bremermann sur la frontière de Silov d'un domaine faiblement pseudoconvexe, C. R. Acad. Sci. Paris Sér. A 279 (1974), A407-A408. MR 50 \# 10321.

2. M. Hakim and N. Sibony, Frontière de Silov et spectre de $A(\bar{D})$ pour des domaines faiblements pseudoconvexes, C. R. Acad. Sci. Paris. Sér. A 281 (1975), A959-A962.

3. K. Hoffman, Banach spaces of analytic functions, Prentice-Hall, Englewood Cliffs, N. J., 1962.

4. J. J. Kohn, Boundary behavior of $\overline{\text { j. }}$ on weakly pseudoconvex manifolds of dimension two, J. Differential Geometry 6 (1972), 523-542. MR 48 \#727.

5. Subellipticity on pseudo-convex domains with isolated degeneracies, Proc. Nat. Acad. Sci. U.S.A. 71 (1974), 2912-2914. MR 50 \#7840.

6. R. M. Range, Bounded holomorphic functions on strictly pseudoconvex domains, Dissertation, Univ. of California, Los Angeles, 1971.

7. W. Rudin, Real and complex analysis, McGraw-Hill, New York, 1966. MR 35 \# 1420.

8. E. M. Stein, Boundary behavior of holomorphic functions of several complex variables, Princeton Univ. Press, Princeton, N. J. 1972.

9. R. O. Wells, Jr., Function theory on differentiable submanifolds, Contributions to Analysis, Academic Press, New York, 1974, 407-441. MR 50 \# 10322.

Department of Mathematics, State University of New York, Albany, New York 12222 